\title{
Dependence of RFID Reader Antenna Design on Read Out Distance
}

\author{
Wim Aerts, Elke De Mulder, Bart Preneel, Guy A. E. Vandenbosch, Senior Member, IEEE, and \\ Ingrid Verbauwhede, Senior Member, IEEE
}

\begin{abstract}
The design of a reader antenna is described for usage in Radio Frequency IDentification (RFID) systems at 13.56 Mhz, as defined in the ISO-14443a standard. It presents the theory, with emphasis on the effect of the read out distance on the design, but also describes measurements on concrete designs to validate the formulas and statements. We also comment on practical problems that were encountered during the design process. The major contribution of this work is the generalization of the design theory for large read out distances where the conventional assumption of constant loop current no longer holds.
\end{abstract}

Index Terms-HF radio communication, loop antennas, magnetic fields, $Q$ factor.

\section{INTRODUCTION}

I SO-14443a 13.56 MHz RFID systems, defined in [1]-[4] use inductive magnetic coupling to set up a two-way communication between a reader and a tag, and to provide the battery-less tag with energy. Therefore, both reader or proximity coupling device (PCD) and tag or proximity IC card (PICC) use a coil as antenna, see Fig. 1. The reader transmits a query to the tag by an amplitude modulated (AM) magnetic field, the tag modulates the load seen by the reader at its coil of the transformer to pass an answer back to the reader.

When Radio Frequency IDentification (RFID) technology became widely adopted, several privacy and security issues came up, which is reflected in publications of, among others, Juels [5] and Langheinrich [6]. The tags designed according to the ISO standard mentioned above are usually "Reader talks first (RTF)" implementations. As a consequence there is a possibility that the tag can be read without the owner being aware of it. This threat is even more dangerous if the distance between tag and reader can be extended. Kfir and Wool [7] and Kirschenbaum and Wool [8] have designed a system for

Manuscript received February 05, 2008; revised September 02, 2008. Current version published December 30, 2008. This work was supported in part by the FWO under project G.0475.05 and by the "Institute for the Promotion of Innovation through Science and Technology in Flanders (IWT-Vlaanderen)."

W. Aerts is with Computer Security and Industrial Cryptography (COSIC), Division of Telecommunications and Microwaves (TELEMIC), Department of Electrical Engineering (ESAT), Katholieke Universiteit Leuven, B-3001 Heverlee, Belgium (e-mail: wim.aerts@esat.kuleuven.be).

E. De Mulder, B. Preneel, and I. Verbauwhede are with Computer Security and Industrial Cryptography (COSIC) Research Lab, Department of Electrical Engineering (ESAT), Katholieke Universiteit Leuven, B-3001 Heverlee, Belgium.

G. A. E. Vandenbosch is with the Division of Telecommunications and Microwaves (TELEMIC), Department of Electrical Engineering (ESAT), Katholieke Universiteit Leuven, B-3001 Heverlee, Belgium.

Digital Object Identifier 10.1109/TAP.2008.2007378
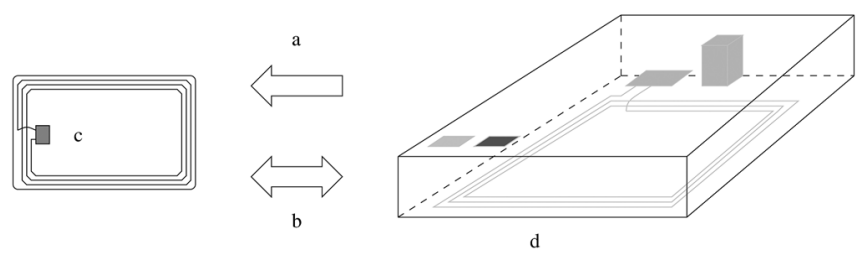

Fig. 1. Communication setup for proximity cards.

relay attacks and a low-cost extended-range RFID skimmer, respectively. In these papers, little focus is put on the actual design of the antenna and the relevant theory to achieve larger read out distances with it. This paper fills the gap. Note that nothing is adjusted to the RFID tag itself as this does not belong to the possibilities of the adversary in this setting.

Here, we first review the relevant theory in Finkenzeller [9] and Yates et al. [10] for designing the reader antenna with a certain activation range. ${ }^{1}$ We extend this and apply it to an ISO-14443a system. The resulting design flow is summarized in Fig. 9. We emphasize the influence of the read out distance, especially if this distance becomes reasonably large, in the order of magnitude of several tens of centimeters. ${ }^{2}$ As most of the commercially available reader systems, such as the MIFARE Pegoda MF RD 700 used in this paper, do not focus on larger reading distance, current enhancement is most likely needed to provide enough current to the coil. Some enhancement techniques are discussed in the paper as they might alter the design decisions.

The antennas discussed in our work are connected to the output of the reader IC. If the antenna has to be placed at a larger distance from the reader hardware itself, a transmission line must be used. This also alters the design procedure, but is taken into consideration as well.

The paper is organized as follows: Section II concentrates on the actual design parameters of the loop antenna. Next several current enhancement techniques are reviewed in Section III as they might have an effect on the design. This is followed by a graphical overview of the design method in Section IV. The paper concludes with a validation in Section V.

\section{LOOP DESIGN}

The reader antenna has to provide the tag with a field that is sufficient to power up the hardware in the tag. Hence, the an-

\footnotetext{
${ }^{1}$ Activation range is defined as the distance from the reader where the field is still large enough to power up the tag.

${ }^{2}$ Even if $10 \mathrm{~cm}$ is often suggested as the maximum range, the ISO-14443 standard does not mention this.
} 
tenna will be designed in such a way that the magnetic field at a certain read out distance $r_{d}$ is large enough. In second order, the antenna should also be suited to receive the answer from the tag. This condition is not considered in the paper, as an adversary is presumed to have access to a separate receiving antenna if needed.

The read out distance can not be infinite, because inductive coupling from the tag to the reader implies that the tag must be in the near field of the reader. Indeed, if the tag receives a traveling wave instead of a quasi-static field, the modifications to the field due to the tag will never travel back to the reader. This means that the tag must be located in the reactive near field [10] around the antenna, in this case $r_{d} \ll \lambda / 2 \pi \approx 4 \mathrm{~m}$. Furthermore, if the total wire length of the loop becomes a considerable part of the wavelength, the loop can not be considered as a lumped element. Standing waves will cause multiple resonances and decrease the total field. In such case $r_{l}$ should be decreased and the current increased even more.

The parameters of the loop that can be chosen are shape, size, number of turns and wire diameter. These are discussed separately later and will lead to a design methodology.

Concerning balun design, needed in case of a balanced-unbalanced transition, the reader is referred to the literature. Baluns can be implemented as $\lambda / 4$ stubs, but at $13.56 \mathrm{MHz}$ these stubs would be impractically long. Another solution would be using transformers [11]. A practical implementation is described in [12]. Yet another solution is to feed the loop in a balanced way, e.g., by a push-pull amplifier instead of a single ended amplifier.

\section{A. Shape}

Of all antennas that can be used to excite a magnetic field, a circular loop is clearly the best choice in case the current on the entire antenna is in phase. As the distance from the point where the tag is located to all current carrying parts of the antenna is equal in this case, the contributions of all parts of the antenna arrive in phase at the tag, resulting in constructive interference.

For a larger loop, where the current over the loop can not be supposed constant, it is less obvious. A spiral can slightly compensate for the phase difference over the loop by a difference in propagation distance to the tag. Moreover, a spiral has a lower inductance $L$ than a circular coil with the same number of turns $N$, resulting in an excellent coupling factor $k$ [13]. Where this may be interesting from a power transfer point of view, it is rather irrelevant for the application envisaged here. When simply looking at maximum attainable magnetic field strength starting from a certain loop current $I_{l}$, the circular loop is still the better choice. Moreover the circular loop outperforms the spiral in case of lateral misalignment [14], which is very likely to occur in case of an adversary secretly reading out tags. As a consequence, the circular loop is the best choice here too.

\section{B. Size}

The larger the circular reader antenna loop is, the more current carrying parts add a contribution to the magnetic field. If the loop becomes too large, however, these contributions are very weak due to the large distance from the current carrying part to the tag. Hence, there will be an optimal loop diameter and this

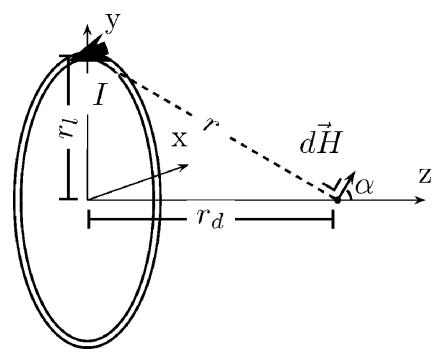

Fig. 2. Reader loop geometry.

diameter is ruled by the read out distance. Suppose that the circular loop has a radius $r_{l}$ and the read out distance is $r_{d}$, then $r_{l}$ should be chosen so that the magnetic field at a distance $r_{d}$ from the center is maximal.

When phase differences ${ }^{3}$ along the total wire length of the loop $\left(2 N \pi r_{l}\right)$ are taken into account, the amplitude of the magnetic field in the direction perpendicular to the tag, at a distance $r_{d}$ becomes (see Fig. 2 for conventions)

$$
\begin{aligned}
\left|\vec{H}_{z}\right| & =\left|\int_{0}^{2 \pi r_{l} N} \frac{I_{l} \exp \left(c \frac{2 \pi l}{\lambda}\right) \cos \alpha}{4 \pi\left(r_{d}^{2}+r_{l}^{2}\right)} d l\right| \\
& =\frac{I_{l} r_{l} \lambda \sqrt{2\left(1-\cos \left(\frac{4 \pi^{2} r_{l}}{\lambda}\right)\right)}}{8 \pi^{2} \sqrt{\left(r_{l}^{2}+r_{d}^{2}\right)^{3}}}\left|\sum_{n=0}^{N-1} e^{j \frac{4 \pi^{2} n r_{l}}{\lambda}}\right|
\end{aligned}
$$

with $N$ the number of turns of the loop, $I_{l}$ the amplitude of the loop current and $\alpha$ the angle indicated in Fig. 2.

Finding the optimal value for $r_{l}$ thus boils down to (numerically) finding the root of the derivative of (1)

$$
\frac{d\left|\vec{H}_{z}\right|}{d \rho}=0 \text { with } \rho=\frac{r_{l}}{r_{d}} .
$$

A general formula for this derivative is given in Appendix. For the case of a single turn $(N=1)$, the optimal value for $\rho$ and, hence, $r_{l}$ is found as the solution of

$$
\frac{\left(2 \rho^{2}-1\right) \lambda}{\rho}-\frac{\sin \left(\frac{4 \pi^{2} \rho r_{d}}{\lambda}\right)}{1-\cos \left(\frac{4 \pi^{2} \rho r_{d}}{\lambda}\right)} 2 \pi^{2} r_{d}\left(1+\rho^{2}\right)=0 .
$$

The result, as well as the solutions for $N=2,3,5,10$, plotted in Fig. 3, show that as $r_{d}$ increases, $r_{l}$ also increases, even up to $r_{d}=4 \mathrm{~m}$. But the ratio between $r_{l}$ and $r_{d}$ decreases as $r_{d}$ increases. For any number of turns, the limit for $r_{d} \rightarrow 0$

$$
\lim _{r_{d} \rightarrow 0} \rho=\lim _{r_{d} \rightarrow 0} \frac{r_{l}}{r_{d}}=\sqrt{2} .
$$

This can be expected as in this limiting case, the assumption of a constant current over the entire wire length of the loop, leading to the ratio $r_{l} / r_{d}=\sqrt{2}$ as published in [9] and [15] surely holds.

1) Total Wire Length of Loop Small Compared to Wavelength: Under this precondition, the current can be assumed

${ }^{3}$ The phase due to the distance between the source current and the tag location is discarded as this distance is the same for all current carrying parts of the circular loop. 


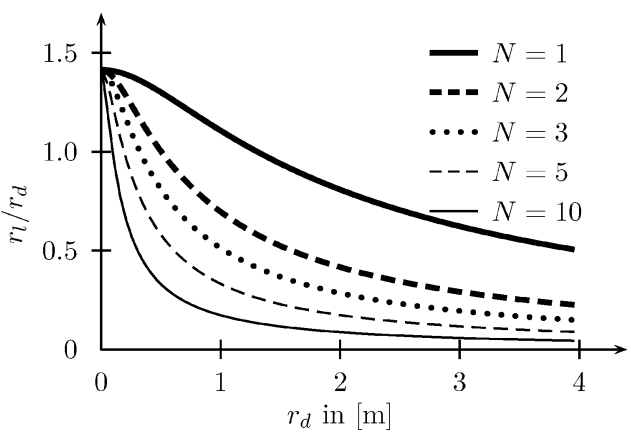

Fig. 3. $r_{l} / r_{d}$ as a function of $r_{d}$.

to be constant over the loop. The field is then found following Choudhury [16]:

$$
\left|\vec{H}_{z}\right|=\left|\int_{0}^{2 \pi r_{l}} \frac{N I_{l} \cos \alpha}{4 \pi r^{2}} d l\right|=\left|\frac{N I_{l} r_{l}^{2}}{2 \sqrt{\left(r_{l}^{2}+r_{d}^{2}\right)^{3}}}\right| .
$$

The ISO-14443 standard [2] specifies the minimum (rms) magnetic field strength for the cards to operate ${ }^{4}$ as $H_{\min }=$ $1.5 \mathrm{~A} / \mathrm{m}$. Combining (5) with (4) yields

$$
\left|\vec{H}_{z}\right|=\left|\frac{N I_{l} r_{l}^{2}}{2 \sqrt{\left(r_{l}^{2}+r_{d}^{2}\right)^{3}}}\right|=\left|\frac{N I_{l} r_{d}^{2}}{\sqrt{\left(3 r_{d}^{2}\right)^{3}}}\right|=\left|\frac{N I_{l}}{\sqrt{27} r_{d}}\right| .
$$

The minimum current (rms) $I_{\min }$ needed in the reader antenna (with $r_{d}$ in $\mathrm{m}$ ) is

$$
I_{\min } \times N=\sqrt{27} \times H_{\min } \times r_{d}=7.8 \times r_{d} .
$$

Assume, e.g., $N=1, r_{d}=0.1 \mathrm{~m}$ and $L=1 \mu \mathrm{H}$. The current with (7) will be $0.78 \mathrm{~A}$ and the voltage

$$
|V|=\left|\omega L \sqrt{27} H_{\min } r_{d}\right| \approx 66 \mathrm{~V}
$$

It is obvious that applying this voltage directly to the loop is not practical. Hence current enhancement techniques, covered in Section III, should be used.

2) Total Wire Length of Loop Comparable to Wavelength: As soon as the total wire length of the loop is considerable, say $\lambda / 10$ as a rule of thumb, the magnetic field of the loop will be smaller than what would be expected when using (5), which is indeed invalid in this case. Due to the phase differences over the loop, the contributions of all parts of the loop at the location of the tag will not be in phase and partial cancellation will occur. In that sense it might even be more advantageous to use a slightly smaller loop with more current, as Fig. 3 indeed indicates.

3) Total Wire Length of Loop Multiple of Half Wavelength: If the loop perimeter equals half a wavelength or a multiple, standing waves will occur. The loop itself will then resonate and the reactance on the Smith chart crosses the real axis, going from inductive to capacitive impedance. This dependence of the reactance on the frequency has its effect on the usage of the coil in an $R L C$ circuit to enhance current. If, for example (16), the resonance condition for a combined series-parallel $R L C$, has, due

\footnotetext{
${ }^{4}$ That is to build up a voltage high enough to power up the hardware in the tag.
}

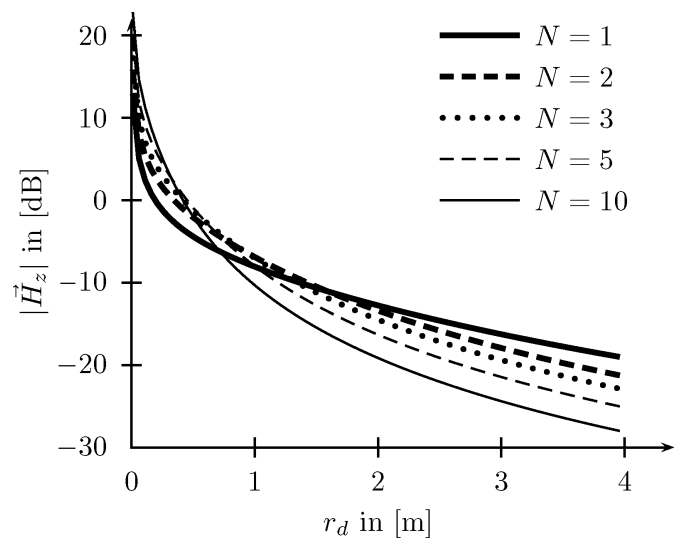

Fig. 4. $\left|\vec{H}_{z}\right|$ at $r_{d}$ when optimal $r_{l}$ for that value of $r_{d}$ is used. $I_{l}=1 \mathrm{~A}$.

to frequency dependence, multiple solutions for different $\omega$, the $R L C$ system will resonate at multiple frequencies and the energy will be divided amongst them. Fig. 8 shows multiple crossings of the curves for the resonance condition and the imaginary part of the input impedance.

\section{Number of Turns $N$}

Looking at (5), which is only valid for small loops and in the case the coil is fed with a current source, it is tempting to think that a high $N$ will result in a high magnetic field $H$ at the tag location. The considerations later, however, argue against a value of $N>1$.

1) Phase Degradation: The more turns are used, the longer the total wire length of the loop and hence the more pronounced the effect described in Section II-B-2 becomes. For a small loop, fed by a current source, the magnetic field can be boosted by taking more turns. But as soon as the total wire length of the loop can not be regarded small compared to the wavelength, it is advantageous to take $N$ smaller. Fig. 4 depicts the magnetic field as a function of $r_{d}$ in the case of $N=1,2,3,5,10$ when the optimal $r_{l}$ as calculated in Section II-B is used. The conclusion that using more turns is only advantageous for smaller $r_{d}$, is clear.

2) Loop Impedance: For a loop, fed by a voltage source, discarding the small ohmic and radiation resistance with respect to $\omega L$, following Schrank and Mahony [17]:

$$
N I_{l}=N \frac{V_{l}}{\omega L}=N \frac{V_{l}}{\omega N^{2} L_{1, N=1}} \propto \frac{V_{l}}{N L_{1, N=1}}
$$

with $L_{1, N=1}$ the inductance of a loop of the same size with only one turn. Thus, maximizing $N I_{l}$, in order to maximize $\left|\vec{H}_{z}\right|$ in (5), means $N=1$ and $L_{1, N=1}$ small.

3) Bandwidth: When the loop antenna is placed in an $R L C$ chain and used for a communication link, raising $N$ is not without a price. As $L$ is proportional to the quality factor, defined later on in (15), it can only be increased up to a certain level as otherwise the bandwidth of the system would become too small. This is not in contradiction with the results of Yates et al. [10], namely that the power transfer ratio is proportional to $N^{2}$, but they do not consider bandwidth issues.

4) Self-Resonance Frequency: When the loop perimeter is about half a wavelength, the loop will resonate. But if the loop 
TABLE I

SELF-Resonance Frequency of Loops With $r_{w}=1 \mathrm{~mm}$ With AND Without InSUlation of $0.2 \mathrm{Mm}$ With $\epsilon_{r}=4$

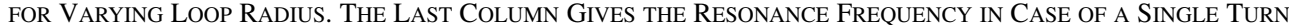

\begin{tabular}{|c|c|c|c|c|c|}
\hline$r_{l}[\mathrm{~m}]$ & $C_{t t}[\mathrm{pF}]$ & $L_{t}[\mu \mathrm{H}]$ & $f_{\text {res }, N=2}\left(\epsilon_{0}\right)[\mathrm{MHz}]$ & $f_{\text {res }, N=2}\left(\epsilon_{r}=4\right)[\mathrm{MHz}]$ & $f_{\text {res, }, N=1}\left(\epsilon_{0}\right)[\mathrm{MHz}]$ \\
\hline 0.01 & 2.016 & 0.07729 & 403.2 & 125.6 & 4775 \\
0.1 & 20.16 & 1.352 & 30.49 & 9.498 & 477.5 \\
1 & 201.6 & 19.3 & 2.551 & 0.7947 & 47.75 \\
\hline
\end{tabular}

has multiple turns, resonance at lower frequency occurs due to the parallel resonance of the inductance of the loop and the capacitance between its turns. Above its resonance frequency, the loop starts to behave as a capacitance. Therefore it is important to know the resonance frequency $f_{\text {res }}$ of the loop.

The capacitance between two turns $C_{\mathrm{tt}}$ can be calculated with Magnusson [18]

$$
C_{\mathrm{tt}}=2 \pi r_{l} \times \frac{\epsilon_{0} \pi}{\log \left(\frac{d}{2 r_{w}}+\sqrt{\left(\frac{d}{2 r_{w}}\right)^{2}-1}\right)}
$$

where $r_{w}$ stands for the wire radius and $d$ is the distance between the centers of the two wires.

If the wire has an insulation with a relative permittivity $\epsilon_{r}$ different from 1, in Grandi [19] the formula becomes

$$
C_{\mathrm{tt}}=2 \pi r_{l} \times \frac{\epsilon_{r} \epsilon_{0} \pi}{\log \left(\frac{d}{2 r_{w}}\right)}
$$

where the insulation of both wires is supposed to touch, resulting in $d=2 r_{w}+2 t$ with $t$ the insulation thickness. This formula only holds for a radial electric field in the insulation, which is surely not the case for $\epsilon_{r} \rightarrow 1$. Hence, substituting $\epsilon_{r}=1$ into formula (11) does not result in (10) due to approximations used in the model that led to (11).

If an inductor with multiple turns is used, the equivalent capacitance is found as the series circuit of all turn-to-turn capacitances. This is a simplification and it assumes that the capacitance between nonadjacent turns can be neglected. In Fig. 5, the equivalent circuit of an inductor with all capacitances is drawn. The capacitances that are neglected are drawn with dashed lines.

Note that discarding the capacitance between nonadjacent turns implies that the self-resonance frequency only shifts downwards by a factor $\sqrt{N}$ when adding more turns. Indeed, the resonance frequency is found as

$$
f_{\mathrm{res}}=\frac{1}{2 \pi \sqrt{L_{1} C_{t t}}}=\frac{1}{2 \pi \sqrt{N L_{1, N=1} C_{t t}}}=\sqrt{\frac{2}{N}} f_{\mathrm{res}, N=2}
$$

where $L_{1, N}$ and $C_{t t}$ indicate the inductance (in the presence of the other turns), respectively, capacitance of a single turn. $L_{1, N}=N \times L_{1, N=1}$ still depends on the number of turns, $L_{1, N=1}$ is the inductance of a single turn in the absence of all other turns. Table I shows the result obtained from (12).

Taking the interturn capacitance into account, the impedance of the coil equals

$$
Z_{\text {coil }}=\frac{j \omega N^{2} L_{1, N=1}}{1-\omega^{2} N L_{1, N=1} C_{t t}} .
$$

which is again frequency dependent, possibly causing resonances at multiple frequencies, similar to those mentioned for $N=1$ in Section II-B-3.
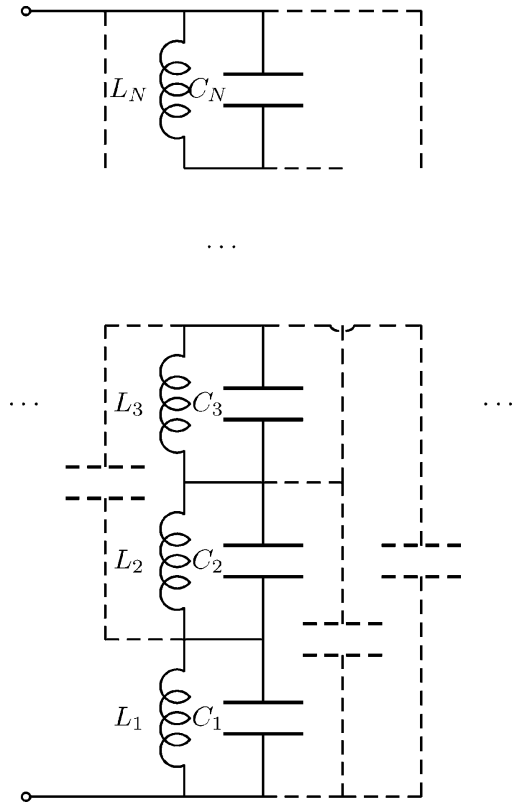

Fig. 5. Equivalent circuit of an inductor.

5) Resistance of Parallel Wires: Another disadvantage of multiturn loops, is their increased ohmic resistance. Due to the interturn coupling, the current in the loop wire is even more confined than should be expected due to the skin effect alone. Smith [20] provides formulas to calculate this effect in the case of a loop that is small compared to the wavelength.

The argumentation above leads to the conclusion that $N$ should be taken small unless only power transfer is considered or the loop antenna is fed by a current source and $r_{d}$ is rather small.

\section{Wire Diameter}

Section II-C revealed that when a voltage source is used, or the loop is placed in an $R L C$ chain and used for data communication, its inductance $L$ should be small. Using a wire with large diameter reduces $L$.

\section{POWER SOURCE AND CURRENT ENHANCEMENT}

As aforementioned and expressed in (7), a minimum amount of current is needed to activate the RFID tag. This current can be directly drawn from an external power source, but sometimes it can be more convenient to enhance the current if for example a powerful power source is not available. This can be done either passively by means of an $R L C$ circuit or actively with the aid of an amplifier. The choice of power source and enhancement technique affects the design of the loop so these need to be taken into consideration. Some passive enhancement techniques are treated later. An active amplifier can enhance the current in the coil even more. A class E amplifier, Sokal and Sokal [21], 

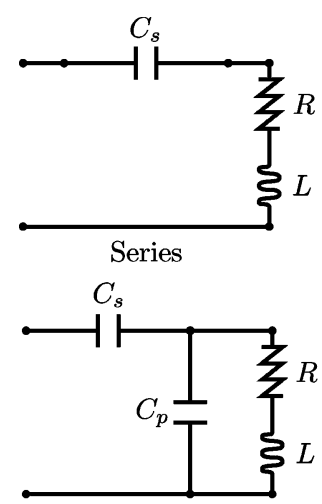

Series-Parallel
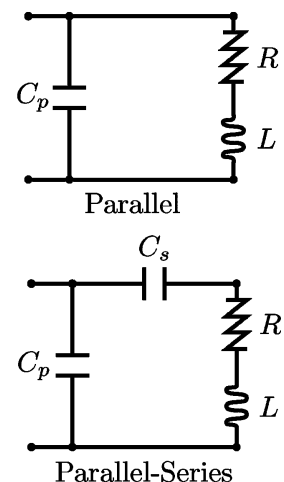

Parallel-Series
Fig. 6. Schematic of (top left) series; (bottom left) combined series-parallel; (top right) parallel; and (bottom right) combined parallel-series $R L C$ resonance circuit.

would, e.g., be a good choice. As such, this is beyond the scope of this paper.

When adding a capacitor and a resistor to the loop, in order to obtain an $R L C$ circuit, many combinations with a capacitor, inductor and resistor can be made. Only the ones with $L$, the inductance of the loop antenna, and $R$ in series are considered for this application, because the loop resistance is inherently in series with the inductance of the antenna [22]. If an external resistor has to be added, it is preferably added in series with the loop, for the same reason. The internal resistance of the capacitors is smaller and will be neglected. An overview of the possible circuits is given in Fig. 6. In this figure the top left configuration is the best choice if a source can deliver an unlimited amount of current, the top right setup is optimal when a source can provide the circuit with high voltage. On the other hand, the bottom configurations ease the need for high voltage or current.

1) Series RLC Chain: In this case, the current in the loop will be maximum when $C_{s}=1 / \omega^{2} L$, the impedance $Z_{\text {series }}=R$ is minimal.

The voltage over the loop is

$$
V_{l}=j \frac{\omega L}{R} V_{\text {source }}=j Q_{R L C} V_{\text {source, }} \text { with } Q_{R L C}=\frac{\omega L}{R}
$$

where $Q_{R L C}$ is the quality factor of the circuit. The higher $Q_{R L C}$, the larger the current in, and the voltage over, the loop, but the lower the bandwidth $B$ of the circuit. This follows from the definitions [23]:

$$
Q_{R L C}=\frac{\text { stored energy }}{\text { dissipated energy per cycle }} \quad \text { and } \quad Q_{R L C}=\frac{f_{0}}{B}
$$

with $f_{0}$ the resonance frequency. As a minimum bandwidth is needed for data transfer, the value for $Q$ is upper bounded by the data rate.

2) Parallel RLC Chain: Here, the current in the loop only depends on the voltage applied to the $R L C$ chain, but the current drawn from the source will be minimum if the condition $C_{p}=$ $L /\left(\omega^{2} L^{2}+R^{2}\right)$ or $Z_{\text {parallel }}=R+\left(\omega^{2} L^{2} / R\right)$ is met. In this case the impedance of the chain is maximal as is the current amplification.
3) Combined Series/Parallel RLC Chain: The loop current is maximized when

$$
\omega^{2} C_{s}=\frac{1-2 \omega^{2} L C_{p}+\omega^{2} C_{p}^{2}\left(\omega^{2} L^{2}+R^{2}\right)}{L-C_{p}\left(\omega^{2} L^{2}+R^{2}\right)} .
$$

Due to the second degree of freedom, $C_{p}$, any value for the impedance can be obtained

$$
Z_{\text {combinedSP }}=\frac{R}{1-2 \omega^{2} L C_{p}+\omega^{2} C_{p}^{2}\left(\omega^{2} L^{2}+R^{2}\right)}
$$

Hence, this is the best choice for the resonance circuit, as it allows to match the internal resistance of any source, to ensure maximum power transfer to the load. If the loop antenna is located at a distance from the reader, a transmission line has to be used to connect both and the use of the combined chain is obligatory: of the four circuits, only this one can match the characteristic impedance of any line.

4) Combined Parallel/Series RLC Chain: The loop current is maximized when $C_{s}=1 / \omega^{2} L$. This is identical to the resonance condition of the series resonance chain. The second degree of freedom, $C_{p}$, can again be used to choose the input impedance of the chain

$$
Z_{\mathrm{combinedPS}}=\frac{R-j \omega R^{2} C_{p}}{1+\omega^{2} R^{2} C_{p}^{2}}
$$

but to a lesser extent than was the case in the series/parallel chain as $Z_{\text {combinedPs }}$ will always be smaller than $R$.

In order to use the $R L C$ equations mentioned earlier, the inductance $L$ of the antenna should be either calculated [24] with

$$
L=\mu_{0} r_{l}\left(\ln \left(\frac{8 r_{l}}{r_{w}}\right)-2\right)
$$

or measured [25]. Both ways of obtaining $L$ are not very accurate. Even more, the value for $L$ also depends on the surroundings, especially for larger loops. The presence of metal is one of the reasons for this alteration. Consequently, the value for the capacitance $C$ needed after installation can differ slightly from the value calculated with the design equations given above. This problem is easily solved by tuning 5 the resonance circuit, using trimming capacitors. Automatic tuning compensates on the fly, but, at the cost of increased complexity. One example [26] uses a control circuit to set the DC bias in a ferrite core to change the inductance of a coil.

The last parameter to determine is the resistance $R$. The total resistance $R$ of the chain will be the internal resistance of the loop $R_{l}$ and an external resistor that is deliberately added. The equations above show that the value of $R$ can also influence the resonance frequency of the chain. This deviation can also be corrected by tuning the capacitors, so that the value for $R$ can be determined only based on the requirements for $Q_{R L C}$.

${ }^{5}$ Try to find the values for the capacitors that result in the largest current in the loop. Monitoring of the current in the loop can be done by: 1) using a current probe, but this adds another inductance; 2) measuring the voltage over the loop, but voltage probes always form a small loop and pick up fields; or 3) using a field probe. The last method is preferred. 
TABLE II

Overview of the Characteristics of the Different Loops, $(*)$ Reading Distance Not Measurable Because of $Q_{R L C}$ Too High,

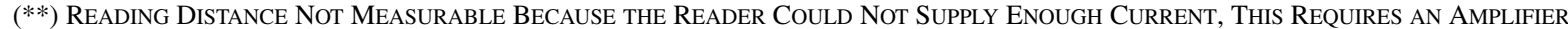

\begin{tabular}{|l||c|c|c|c|c|c|c|c|c||c|c|}
\hline \multicolumn{1}{|c||}{} & \multicolumn{9}{c||}{ coil } & \multicolumn{3}{c|}{ RLC circuit } \\
\cline { 2 - 10 } & $N$ & $\begin{array}{c}r_{l} \\
{[\mathrm{~cm}]}\end{array}$ & $\begin{array}{c}d \\
{[\mathrm{~cm}]}\end{array}$ & $\begin{array}{c}R_{l} \\
{[\Omega]}\end{array}$ & $\begin{array}{c}L_{\text {calc }} \\
{[\mu \mathrm{H}]}\end{array}$ & $\begin{array}{c}L_{\text {meas }} \\
{[\mu \mathrm{H}]}\end{array}$ & $\begin{array}{c}f_{\text {res,calc }}[\mathrm{MHz}] \\
{\left[\begin{array}{c}f_{\text {res,meas }} \\
{[\mathrm{MHz}]}\end{array}\right.}\end{array}$ & $\begin{array}{c}H_{(x y z=0)} \\
{[\mathrm{A} / \mathrm{m}]}\end{array}$ & $\begin{array}{c}r_{d} \\
{[\mathrm{~cm}]}\end{array}$ \\
\hline Solid copper wire loop 1 & 1 & 8.25 & 0.1 & 0.5 & 0.537 & 0.569 & 579 & 583 & 4.16 & 10 \\
Solid copper wire loop 2 & 2 & 8.25 & 0.1 & 2.09 & 2.15 & 2.27 & 48 & 41 & 5.35 & $(*)$ \\
Copper tube loop & 1 & 56.5 & 1.5 & 200 & 3.1 & 3.45 & 85 & 27 & 0.12 & $(* *)$ \\
\hline
\end{tabular}

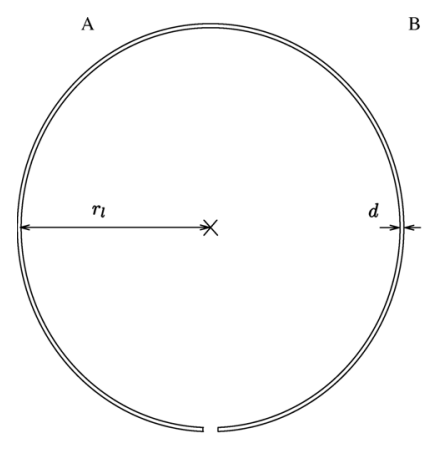

(a)

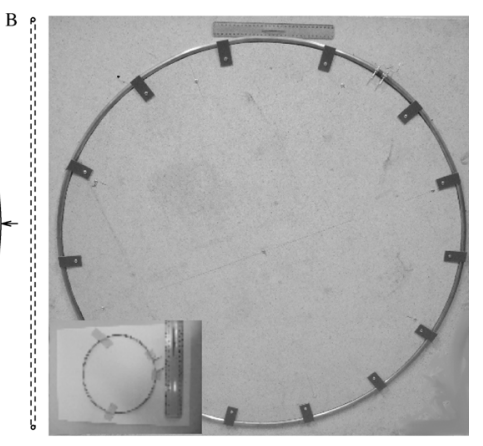

(b)

Fig. 7. Layout drawing and pictures of loops designed and made to validate the formulas and statements. In the schematic drawing (a): $\mathrm{A}=$ front view, $\mathrm{B}=$ cross section. The values for $r_{l}$ and $d$ of the different antennas can be found in Table II. $N=1$. The same schematic holds for $N>1$, only more turns are stacked. (b) shows the largest loop made (the copper tube loop) and the smaller two turn loop made from solid copper.

\section{DESIGN FlOWCHART}

An overview of the proposed design method is given in Fig. 9. The reading distance $r_{d}$ and the working frequency $f$ result with the aid of the derivative of formula (1) in the ideal loop radius $r_{l}$. The source and the quality factor $Q_{\text {system }}$ determine the number of turns, $N$, according to Section II-C. When choosing $N$, the self-resonance of the loop should be checked to ensure that the self-resonance frequency of the loop is higher than the working frequency $f$. The loop radius is another parameter which affects this self-resonance. The specifications of the application also define $H_{\min }$. From this, together with $f$ and $r_{d}$, it is possible to calculate $I_{\min }$, see (7).

If current enhancement is needed, a decision on the type of passive enhancement can be taken based on the type of source. Together with the inductance of the loop $L$ and the internal resistance $R_{l}$, which are defined by a number of characteristics of the loop, the needed $R$ and $C$ for the $R L C$-circuit are easily found by applying the formulas in Section III. After some additional manual tuning, the design is finished.

If no current enhancement is needed, the circuit should be checked for unwanted resonances; if these are present, suppression should be supplied.

\section{VALIDATION AND CONCLUSION}

Three antennas were made to validate the formulas and statements: two solid copper wire loops, one with $N=1$, another one with $N=2$ and one copper tube loop, see Fig. 7. Their geometrical parameters are summarized in Table II.

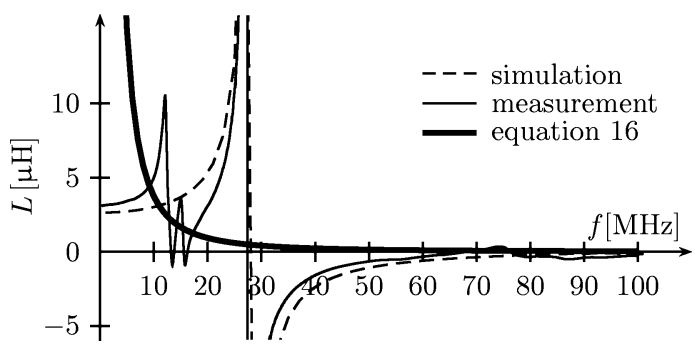

Fig. 8. Frequency dependent value of $L$ for the copper tube loop as obtained from simulation and measurement. A balanced to unbalanced system transition causes this value to fluctuate heavily around $13 \mathrm{MHz}$ so that the loop is useless unless fed in a balanced way.

The MIFARE Pegoda MF RD 700 is used as a reader. Its RF output is a voltage source with internal resistance. For such a source, as explained in Section III, the best choice is the series-parallel circuit. Tuning is necessary because the frequency response of the circuit is very sensitive to $L$ and neither the measured $L_{\text {meas }}$ nor the calculated $L_{\text {calc }}$ are accurate enough. The calculated inductances $L_{\text {calc }}$ were verified against values derived from measurements. Due to balun problems the measurements were inaccurate for the copper tube loop; instead the loop was replaced by lumped elements until the resonance frequency matched the original one. The values obtained can be found in Table II.

The resonance circuit is a variation on the series-parallel circuit: it consists of an upright and a mirrored version of it to feed the antenna in a balanced way. If this is not done, problems as those shown in Fig. 8 can arise because of a transition from a balanced loop to an unbalanced vectorial network analyzer. The simulated curve is obtained from a NEC simulation with 400 divisions along the circle perimeter.

Furthermore, Table II also lists some electrical parameters: the magnetic field $H$ at the origin of the loop and the maximum reading distance with a MIFARE card. For the latter measurement no other alterations were done to the setup but tuning the resonance circuit. For the first measurement, an EMCO - 902, 3-cm magnetic field probe is utilized [27].

In Table II, it is noticed that the loop with two turns generates a higher magnetic field compared to the loop with one turn, but this does not result in a larger reading distance because of a $Q_{R L C}$ which is too high, so that even at a very small distance no communication can take place in this setup. Adding an external resistor can solve this, but this is beyond the scope of validating the formulas and statements. When holding a card very close to the copper tube, this card could be read by the reader, but the reader could not supply enough current to obtain a functioning system with a card at the origin or further along the $z$ axis. 


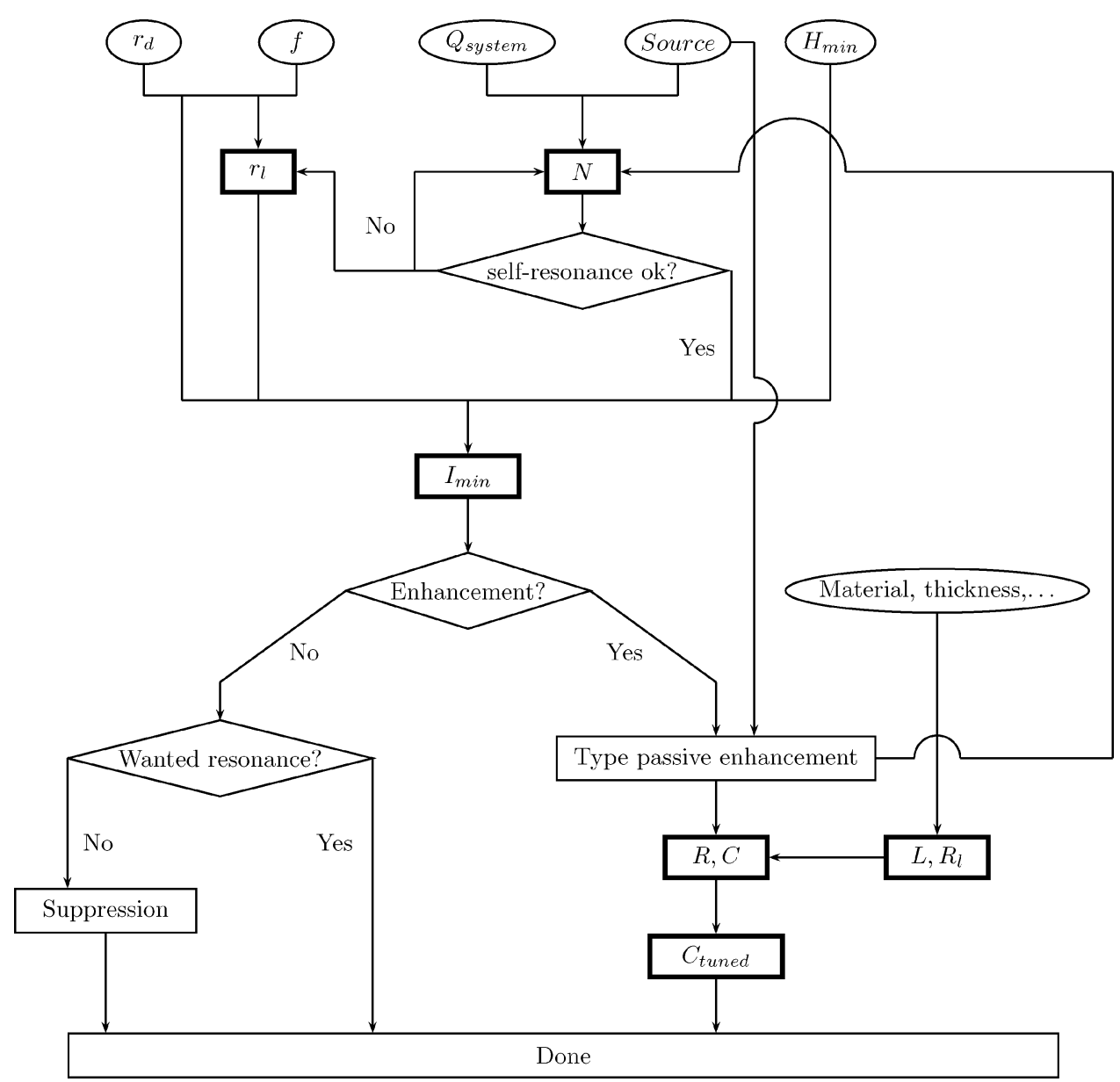

Fig. 9. Flowchart of the design method.

Another difference between the two solid wire loops is the remarkably lower $f_{\text {res }}$ of the two turn loop. This confirms the explanation of (11) where the effect of $C_{t t}$ is demonstrated. Here, $\epsilon_{r}=4$ [28].

From these measurement results the conclusion can be drawn that the formulas to design a loop antenna for an ISO-14443 system summarized in this paper, are valid.

\section{APPENDIX}

\section{GeNeral Formula FOR THE DERIVATIVE OF (1)}

Writing (1) as the product of two functions $f(\rho) g(\rho)$ and leaving out all constants, because of no importance for the derivative, results in

$$
\begin{aligned}
f(\rho) & =\frac{\rho}{\left(1+\rho^{2}\right)^{\frac{3}{2}}} \sqrt{1-\cos \left(\frac{4 \pi^{2} \rho r_{d}}{\lambda}\right)} \\
g(\rho) & =\left|\sum_{n=0}^{N-1} e^{j \frac{4 \pi^{2} n r_{l}}{\lambda}}\right| \\
& =2 \times\left(\sum_{n=0}^{N}(N-n) \cos \left(n \frac{4 \pi^{2} \rho r_{d}}{\lambda}\right)\right)-N .
\end{aligned}
$$

The derivative of those functions is

$$
\begin{aligned}
f^{\prime}(\rho)= & \frac{\sqrt{1-\cos \left(\frac{4 \pi^{2} \rho r_{d}}{\lambda}\right)}\left(1-2 \rho^{2}\right)}{\left(1+\rho^{2}\right)^{\frac{5}{2}}}\left(\frac{2 \pi^{2} \rho r_{d} \sin \left(\frac{4 \pi^{2} \rho r_{d}}{\lambda}\right)}{\lambda\left(1+\rho^{2}\right)^{\frac{3}{2}} \sqrt{1-\cos \left(\frac{4 \pi^{2} \rho r_{d}}{\lambda}\right)}}\right. \\
& +\frac{4 \pi^{2} r_{d}}{\lambda} \sin \left(n \frac{4 \pi^{2} \rho r_{d}}{\lambda}\right) .
\end{aligned}
$$

Knowing that

$$
(f g)^{\prime}=f^{\prime} g+f g^{\prime}
$$

the equation to be solved in order to find the optimal $\rho$, is

$$
\begin{aligned}
& \left(\left(1-\cos \left(\frac{4 \pi^{2} \rho r_{d}}{\lambda}\right)\right) \lambda\left(1-2 \rho^{2}\right)\right. \\
& \left.\quad+2 \pi^{2} \rho r_{d}\left(1+\rho^{2}\right) \sin \left(\frac{4 \pi^{2} \rho r_{d}}{\lambda}\right)\right) \\
& \quad \times\left(2\left(\sum_{n=0}^{N}(N-n) \cos \left(n \frac{4 \pi^{2} \rho r_{d}}{\lambda}\right)\right)-N\right)
\end{aligned}
$$




$$
\begin{aligned}
& -2 \rho\left(1+\rho^{2}\right)\left(1-\cos \left(\frac{4 \pi^{2} \rho r_{d}}{\lambda}\right)\right) \\
& \times\left(\sum_{n=0}^{N} n(N-n) 4 \pi^{2} r_{d} \sin \left(n \frac{4 \pi^{2} \rho r_{d}}{\lambda}\right)\right)=0 .
\end{aligned}
$$

\section{REFERENCES}

[1] Identification Cards-Contactless Integrated Circuit(s) Cards-Proximity Cards-Part 1: Physical Characteristics, Int. Std. ISO/IEC 14443-1, ISO/IEC/JTC1 Inf. Technol., 1997, ISO/IEC/JTC1.

[2] Identification Cards-Contactless Integrated Circuit(s) Cards-Proximity Cards-Part 2: Radio Frequency Power and Signal Interface, Int. Std. ISO/IEC 14443-2, ISO/IEC/JTC1 Inf. Technol., 1999, ISO/IEC/ JTC1.

[3] Identification Cards-Contactless Integrated Circuit(s) Cards-Proximity Cards—Part 3: Initialization and Anticollision, Int. Std. ISO/IEC 14443-3, ISO/IEC/JTC1 Inf. Technol., 1999, ISO/IEC/JTC1.

[4] Identification Cards-Contactless Integrated Circuit(s) Cards-Proximity Cards-Part 4: Transmission Protocol, Int. Std. ISO/IEC 14443-4, ISO/IEC/JTC1 Inf. Technol., 2000, ISO/IEC/JTC1.

[5] A. Juels, "RFID security and privacy: A research survey," IEEE J. Sel. Areas Commun., vol. 24, no. 2, pp. 381-394, Feb. 2006.

[6] M. Langheinrich, "RFID and privacy," in Security, Privacy, and Trust in Modern Data Management, M. Petkovic and W. Jonker, Eds. Berlin Heidelberg, New York: Springer, Jul. 2007, ch. 28, pp. 433-450.

[7] Z. Kfir and A. Wool, Picking Virtual Pockets Using Relay Attacks on Contactless Smartcard Systems 2005 [Online]. Available: citeseer.ist. psu.edu/kfir05picking.html

[8] I. Kirschenbaum and A. Wool, "How to build a low-cost, extended-range RFID skimmer," in Proc. 15th USENIX Security Symp., Jul. 13-Aug. 4 2006, pp. 43-57.

[9] K. Finkenzeller, RFID Handbook, 2nd ed. New York: Wiley, 2003.

[10] D. C. Yates, A. S. Holmes, and A. J. Burdett, "Optimal transmission frequency for ultralow-power short-range radio links," IEEE Trans. Circuits Syst., vol. 51, no. 7, pp. 1405-1413, Jul. 2004.

[11] R. W. Lewallen, W7EL, "Baluns: What they do and how they do it," in ARRL Antenna Compendium pp. 157-164, 1985.

[12] HF Antenna Cookbook-Technical application report-radio frequency identification systems Texas Instruments, 2004, Tech. Rep..

[13] C. M. Zierhofer and E. S. Hochmair, "Geometric approach for coupling enhancement of magnetically coupled coils," IEEE Trans. Biomed. Eng., vol. 43, no. 7, pp. 708-714, Jul. 1996.

[14] K. Fotopoulou and B. W. Flynn, "Optimum antenna coil structure for inductive powering of passive RFID tags," in Proc. IEEE Int. Conf. RFID, 2007, pp. 71-77.

[15] Y. Lee, "RFID coil design," in Microchip Technol. Inc. Appl. Note 1998.

[16] M. H. Choudhury, Electromagnetism, J. W. Mason, Ed. Chichester and London, U.K.: Ellis Horwood, 1989.

[17] H. Schrank and J. D. Mahony, "Approximations to the radiation resistance and directivity of circular-loop antennas," IEEE Antennas Propag. Mag., vol. 36, no. 4, pp. 52-55, Aug. 1994.

[18] P. C. Magnusson, G. C. Alexander, V. K. Tripathi, and A. Weisshaar, Transmission Lines and Wave Propagation. Boca Raton, FL: CRC, 2001.

[19] A. M. Gabriele Grandi, M. K. Kazimierczuk, and U. Reggiani, "Stray capacitances of single-layer solenoid air-core inductors," IEEE Trans. Ind. Appl., vol. 35, no. 5, pp. 1162-1168, Sep./Oct. 1999.

[20] G. S. Smith, "Radiation efficiency of electrically small multiturn loop antennas," IEEE Trans. Antennas Propag., Sep. 1972.

[21] N. O. Sokal and A. D. Sokal, "Class E-A new class of high-efficiency tuned single-ended switching power amplifiers," IEEE J. Solid-State Circuits, vol. SC-10, no. 3, pp. 168-176, Jun. 1975.

[22] D. J. Goedbloed, Elektromagnetische compatibiliteit Ten Hagen en Stam, Den Haag/Deventer, 2000.

[23] D. Staelin, A. Morgenthaler, and J. Kong, Electromagnetic Waves. New York: Prentice-Hall Int., 1994.

[24] F. W. Gover, Inductance Calculations: Working Formulas and Tables. New York: Dover, 1946.

[25] S. Harris, "An extension of the method of measuring inductances and capacities," Proc. IRE, vol. 17, no. 3, pp. 516-518, Mar. 1929.

[26] G. Steiner, H. Zangl, P. Fulmek, and G. Brasseur, "A tuning transformer for automatic adjustment of resonant loop antennas in RFID systems," in Proc. IEEE Conf. Ind. Technol. (ICIT), 2004.
[27] “Users's Manual Near-Field Probe Set Model 7405” EMC Test Syst. (ETS) [Online]. Available: http://www.emctest.com, Jan. 1999, E PN 399107 ed., ETS

[28] I. Diaconu and D. Dorohoi, "Properties of polyurethane thin films," Optoelectron. Adv. Mater., vol. 7, no. 2, pp. 921-924, Apr. 2005.

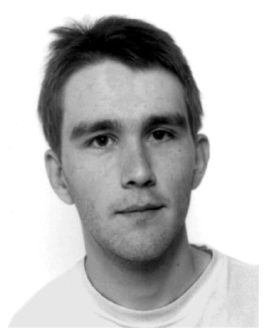

Wim Aerts was born in 1978 in Wilrijk, Belgium. He received the M.S. degree from the Katholieke Universiteit Leuven, Belgium, in 2001, with a thesis on the decoding of tailbiting codes

$\mathrm{He}$ joined the Division of Telecommunications and Microwaves (TELEMIC), Katholieke Universiteit Leuven, Heverlee, Belgium, in August 2001 where he worked on electronically steerable arrays for LEO satellite applications. In July 2007, he joined the Computer Security and Industrial Cryptography (COSIC), Katholieke Universiteit Leuven, where he is working toward the Ph.D. degree by applying array antennas in EM side-channel analysis.

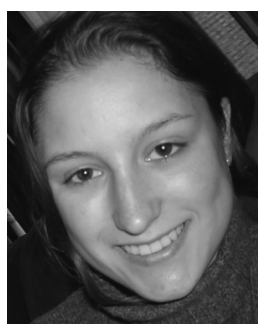

Elke De Mulder was born in Duffel, Belgium, on January 27,1981 . She received the M.Sc. degree in electrical engineering from the Katholieke Universiteit Leuven, Belgium, in July 2004.

She then joined Computer Security and Industrial Cryptography (COSIC), Katholieke Universiteit Leuven, where she is pursuing the Ph.D. degree by working on the topic of "ElectroMagnetic Analysis (EMA)" under supervision of Prof. Ingrid Verbauwhede and Prof. Bart Preneel.

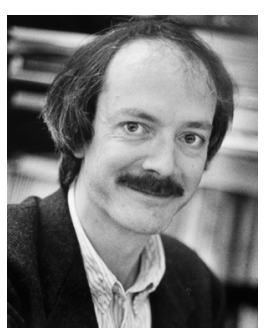

Bart Preneel received the M.S. degree in electrical engineering and the Ph.D. degree in applied sciences (cryptology) from the Katholieke Universiteit Leuven, Belgium, in 1987 and 1993, respectively.

He is currently Full Professor with the Katholieke Universiteit Leuven. He was Visiting Professor at five universities in Europe and was a Research Fellow with the University of California at Berkeley. He has authored and coauthored more than 200 reviewed scientific publications and is the inventor of two patents. His main research interests are cryptography and information security.

Prof. Preneel is President of the International Association for Cryptologic Research (IACR) and of the Leuven Security Excellence Consortium (L-SEC vzw.), an association of 60 companies and research institutions in the area of e-security. He is a member of the Editorial Board of the Journal of Cryptology, the IEEE TRANSACTIONS ON FORENSICS AND INFORMATION SECURITY, and the International Journal of Information and Computer Security. He has participated in more than 20 research projects sponsored by the European Commission, for four of these as Project Manager. He has been Program Chair of 10 international conferences (including Eurocrypt 2000, SAC 2005, and ISC 2006) and he has been Invited Speaker at more than 30 conferences. In 2003, he received the European Information Security Award in the area of academic research. He also received an honorary Certified Information Security Manager (CISM) designation by the Information Systems Audit and Control Association (ISACA).

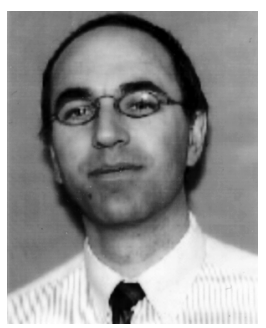

Guy Vandenbosch (M'85-SM'08) was born in SintNiklaas, Belgium, on May 4, 1962. He received the M.S. and Ph.D. degrees in electrical engineering from the Katholieke Universiteit Leuven, Belgium, in 1985 and 1991, respectively. He holds a certificate of the postacademic course in Electro-Magnetic Compatibility from the Technical University Eindhoven, The Netherlands.

He was a research and teaching assistant from 1985 to 1991 with the Telecommunications and Microwaves section of the Katholieke Universiteit Leuven, where he worked on the modeling of microstrip antennas with the 
integral equation technique. From 1991 to 1993, he held a postdoctoral research position with the Katholieke Universiteit Leuven. Since 1993, he has been a Lecturer, and since 2005, a Full Professor at the same university. He teaches courses on "Wireless and Mobile Communications, Part Antennas," "Digital Steer- and Measuring Techniques in Physics," and "Electromagnetic Compatibility." His research interests are in the area of electromagnetic theory, computational electromagnetics, planar antennas and circuits, electromagnetic radiation, electromagnetic compatibility, and bioelectromagnetics. His work has been published in approximately 85 papers in international journals and has been presented at about 140 international conferences.

Dr. Vandenbosch has convened and chaired numerous sessions at many conferences. He was Co-Chairman of the European Microwave Week 2004 in Amsterdam, The Netherlands, and chaired the TPC of the European Microwave Conference within this week. He was a member of the TPC of the European Microwave Conference in 2005, 2006, 2007, and 2008. He has been a member of the "Management Committees" of the Consecutive European COST Actions on Antennas since 1993, where he is leading the working group on modeling and software for antennas. Within the ACE Network of Excellence of the EU (2004-2007), he was a member of the Executive Board and coordinated the activity on the creation of a European antenna software platform. Since 2001, he has been President of SITEL, the Belgian Society of Engineers in Telecommunication and Electronics. Since 2008, he has been a member of the board of FITCE Belgium, the Belgian branch of the Federation of Telecommunications Engineers of the European Union. During 1999-2004, he was Vice Chairman of the IEEE Benelux Chapter on Antennas en Propagation and currently holds the position of Secretary of this Chapter. During 2002-2004, he was Secretary of the IEEE Benelux Chapter on EMC.

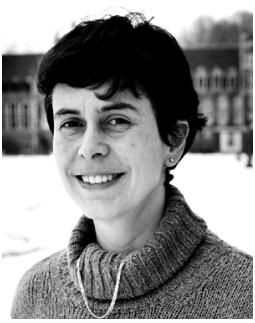

Ingrid Verbauwhede (M'92-SM'02) received both the electrical engineering and $\mathrm{Ph}$.D. degrees from the Katholieke Universiteit Leuven, Belgium, in 1991.

From 1992 to 1994, she was a Postdoctoral Researcher and Visiting Lecturer with the Electrical Engineering and Computer Science Department, University of California at Berkeley (UC Berkeley). From 1994 to 1998, she was with TCSI and ATMEL, Berkeley. In 1998, she joined the faculty of the University of California at Los Angeles (UCLA). She is currently a Professor with the Katholieke Universiteit Leuven, and an adjunct professor with UCLA. Her interests include circuits, processor architectures, and design methodologies for real-time embedded systems for security, cryptography, digital signal processing, and wireless communications. This includes the influence of new technologies and new circuit solutions on the design of next generation systems-on-chip.

Dr. Verbauwhede was the Program Chair of the 2007 Cryptographic Hardware and Embedded Systems (CHES) conference, the 2008 Application-specific Systems, Architectures and Processors (ASAP) conference, the 2002 ACM/IEEE International Symposium on Low Power Electronics and Design symposium (ISLPED). She was also the General Chair of the 2003 ISLPED symposium. She was a member of the executive committee of the $42 \mathrm{nd}$ and 43th Design Automation Conference (DAC) as the Design Community Chair. 